Trials

\begin{tabular}{lrrrrrrrrrr}
\cline { 2 - 8 } Type of Cluster & 1 & 2 & 3 & 4 & 5 & 6 & 7 & 8 & 9 \\
\hline Contiguous & 3.10 & 2.05 & 2.35 & 2.05 & 2.35 & 2.40 & 2.35 & 2.95 & 2.70 \\
Noncontiguous & .25 & .40 & .25 & .55 & .70 & .85 & .85 & .65 & .85 \\
\hline
\end{tabular}

The Ss were 20 male and female undergraduates who were fulfilling a course requirement in introductory psychclogy. Ten Ss were assigned randomly to VD List $A$, and the remaining received List $B$.

RESULTS AND DISCUSSION

The mean number of trials to criterion on the VD list was 6.95. FR protocols were scored by counting the number of contiguous and noncontiguous clusters recalled. A contiguous cluster was defined as recall of the two members of a VD pair in succession. Noncontiguous clusters were VD pairs from List $B$, if $S$ practiced List $A$ on the VD task, or VD pairs from List $A$, if $S$ received List B in VD learning. Since pairs which were noncontiguous for half the Ss were contiguous pairs for the other half of the Ss, amount of noncontiguous pair clustering gives an empirical estimate of clustering attributable to chance and to other factors not related to the contiguous presentation of the members of a pair on the VD task.

The total number of contiguous clusters for Ss in the List A subgroup was 206; this value for the List $B$ subgroup was 240 . These scores do not differ significantly $(t=.55)$. The total number of noncontiguous clusters was 50 and 57 for List A and List B subgroups, respectively. These latter scores are also not reliably different $(t=.46)$. In view of the above, the two subgroups were combined for further analyses. The mean numbers of the two types of clusters over the course of FR learning are presented in Table 1. More contiguous than noncontiguous clusters were recalled. A randomized block factorial analysis of variance indicated that this effect was highly significant, $F(1,323)=150.36, \quad p<.001$. The effect of trials was not significant, $F<1$. Also, the interaction of cluster type with trials fell far short of significance, $\mathrm{F}<1$.

The mean number of words recalled, of course, increased over trials. The nine means for Trials 1.9 were: $10.85,13.05,15.55,16.65$, $17.45,18.75,18.90,20.50$, and 20.35 . Some views of memorial organization suggest that degree of organization should be positively correlated with amount recalled. In this experiment the correlation between contiguous clustering and total recall, while

positive, was far from significant $[r(18)=.21, p>.10]$.

The present data indicate that the contiguous presentation of unrelated words during VD learning induced Ss to recall the words together on the FR task. Wallace's (1970) emphasis on the principle of contiguity as a factor in organization of recall is consistent with these results.

The organization obtained in this experiment can be considered associative clustering (see above) where the clustering of the preexperimentally unrelated items is attributable to the contiguous exposure of the items prior to FR learning. Wallace (1969) has recently obtained laboratory induced clustering when unrelated words wery presented successively during the study trial in a modified FR experiment. In sum, the present data, together with Wallace's, suggest that organization of recall

\title{
Stimulus alternation and
}

continuous short-term memory
in young children*

\section{F. MICHAEL RABINOWITZ†, Tulane University, New Orleans, La. 70118 \\ DAVID B. KRONFIELD, University of Washington, Seattle, Wash. 98105 and}

JOSEPH C. CAMPIONE, University of Illinois, Urbana, Ill. 61820

The effect of the number of interpolated trials on the stimulus alternation behavior of kindergarten children was investigated in a free-choice task. Three sets of stimuli were used for both interpolation and test trials. Stimulus alternation decreased markedly from 0 to 1 interpolated trial and then remained relatively constant, but significantly above chance, over 1,2 , and 3 interpolated trials. The relevance of the findings to stimulus alternation theory and the study of continuous short-term memory in young children was discussed.

In a number of recent experiments (Harris, 1965, 1967; Rabinowitz \& DeMyer, in press), evidence was obtained indicating that children between 4 and 6 years old

* The research was conducted in the Shoreline School District, Seattle, Washington, The authors are extremely grateful for the cooperation they received from teachers and administrators in the Shoreline System. Special thanks are due to Stanley Wiklund. Principal of Ridgecrest Elementary School, William Randall, the Director of Research, and Daniel Marken, Research Associate.

tRequests for reprints should be sent to F. Michael Rabinowitz. Psychology, Newcomb College, Tulane University New Orleans, La. 70118 alternate stimuli. In order to explain this phenomenon, it is necessary to postulate some trace on Trial $n+1$ of the events occurring on Trial n (see Dember \& Fowler, 1958). Whatever the nature of the trace, it should be susceptible to interference. The present experiment was designed in order to: (1) investigate the deterioration rate of trace elements used by young children when they alternate stimuli in a situation in which both proactive and retroactive interference occur; and (2) develop a technique that is useful in studying continuous short-term memory in young children. 


\section{SUBJECTS}

The Ss were 32 kindergarten children, 16 boys and 16 girls. Their ages ranged from 67 to 78 months. Three additional $S$ s were run but did not meet one or more of the criteria outlined below.

\section{STIMULI}

Three sets of two blocks each were the stimuli. Each block had a surface area of approximately $100 \mathrm{sq}$ in and was cut from 3/8-in. plywood. The blocks were painted with glossy paint. Set $1(2,3)$ consisted of a black and a yellow equilateral triangle (a green and a grey square, a blue and a white circle).

\section{DESIGN AND PROCEDURE}

All Ss experienced two experimental sessions, usually separated by a 2-day interval, although longer intervals did occur. In each session, $\mathrm{S}$ was seen individually and was given a series of 16 trials. The block sets were presented in the following order: $A, A_{0}, B, C, A_{2}, B_{2}$, $A_{1}, C_{3}, C_{0}, B_{3}, A_{3}, C=B, C_{1}, B_{1}$, $B_{0}$. The letters represent the block set presented on the trial. Subscripts designate test trials, with the actual subscript indicating the number of trials interpolated between the previous presentation of that set and the test trial. For example, the $\mathrm{A}_{2}$ occurring in Position 5 indicates that Block Set A was presented on the fifth trial, that this was a test trial, and that two trials had intervened since the previous presentation of Set $A$.

Two groups of block sets were constructed in a modified random fashion so that the sets designated $A$, $B$, and $C$ were different in each group. Half the boys and half the girls were assigned to each group. The spatial arrangement of the blocks on each trial was determined randomly subject to the restrictions that, as nearly as possible, across the 2 days: (1) each set appear in each spatial arrangement an equal number of times; and (2) within each of the interpolation conditions, the arrangement of the blocks be equally likely to be the same or the reverse of the arrangement on the previous presentation of that set. A different series was used on Days 1 and 2, with position series being confounded with days. In summary, the variables of interest in the experiment were sex, block sets, day (confounded with position series), and, most important, number of interpolated trials.

The instructions and manner of presenting the blocks were modeled after those used by Harris (1965). Each $S$ was brought from his kindergarten class to a small room in a trailer which was parked on school grounds. The $\mathrm{S}$ and $\mathrm{E}$ were seated on opposite sides of a table, with the blocks hidden from S's view in a box.
The E said, "I have some toys to show you," then picked up the two blocks constituting the appropriate block set and held them, one in each hand, before $S$ and said, "Which one do you want? This one? [E extended one block, then retracted it.] Or this one? $[\mathrm{E}$ extended the other block, then retracted it.]" During this part of the procedure, $\mathrm{E}$ would present the block and wait until $S$ looked at it before retracting it. The hand extended first was counterbalanced across each $\mathrm{S}$. After $S$ had been exposed to each block, both blocks were placed in front of $S$, and $S$ was allowed to choose one of the blocks to play with for $20 \mathrm{sec}$. After the allotted time period, $E$ requested that $S$ return the block. The block was removed from S's view, and the next trial was initiated in the same manner approximately $7 \mathrm{sec}$ later, the time required for the removal and representation of the blocks. T'he time between successive trial presentations was about 30 sec. A stopwatch was used in controlling the various time intervals.

Since the deterioration rate of trace elements used by children when they alternate stimuli was of interest here, an attempt was made to eliminate Ss whose responses were determined by either stimulus or position preferences rather than stimulus alternation. Accordingly, Ss were excluded from the analysis if: (1) they alternated stimuli on less than 12 of the 24 test trials (one $S$ ); or (2) if they responded to the same side on 26 or more of the 32 trials (two $\mathrm{Ss}$ ).

RESULTS AND DISCUSSION

The dependent variable used in the analysis of variance was the number of times $S$ alternated stimuli at each of the interpolation lengths $(0,1,2$, and 3) each day. An S alternated stimuli on a given trial if he chose a different block (e.g., black triangle) than he chose on the prior trial with the same set (e.g., yellow triangle). The number of stimulus alternations for each interpolation length per day could range between 0 and 3 . The between-Ss factors in the analysis of variance were sex and group of block sets, while the within-Ss variables were days and interpolation length. The only significant contrast effect was the main effect of interpolation length $(F=15.67, \mathrm{df}=3 / 84, \mathrm{p}<.001)$. The mean percentages of stimulus alternation averaged across sex, groups of block sets, and days for $0,1,2$, and 3 interpolations were $90.6 \%, 70.3 \%$, $60.4 \%$, and $61.6 \%$, respectively. Scheffé (1959) follow-up tests revealed that the number of stimulus alternations after 1,2 , and 3 interpolations did not differ significantly, while the number of alternations occurring after 0 interpolations was significantly greater than that averaged across 1,2 , and 3 interpolations $\left(\mathrm{S}^{2}=42.48, \mathrm{df}=3 / 84\right.$, $\mathbf{p}<.001$ ). It is important to note, however, that Ss were still alternating stimuli significantly above the chance level even at 2 and 3 interpolations $(\mathrm{z}=2.04$ and 2.35 , respectively, normal approximation to the binomial test). Two of the interaction effects approached significance, the Block Sets by Interpolation Length and the Sex by Days by Interpolation Length ( $p<.10$ in both cases), but their importance seems minimal.

The findings are consistent with the notion that the deterioration rate of trace elements that young children utilize in spontaneous stimulus alternation is negatively accelerated under conditions in which both proactive and retroactive interference are present. The rapid drop in stimulus alternation from 0 to 1 interpolation is consistent with the retention loss reported by Peterson, Saltzman, Hillner, \& Land (1962) with adult Ss in a paired-associates task. Interestingly, the children in the present study still demonstrated above-chance alternation after three interpolated trials. Just how long young children can retain some information about prior block sets under the present conditions can be answered with future experimental work. The technique would also seem to be useful in investigating additional questions concerning young children's continuous short-term memory. For example, the number of stimulus sets used in testing could be systematically varied and the effects noted. The most striking advantage of the technique is that no special training procedures need to be utilized in assessing continuous short-term memory. However, there are also some obvious disadvantages. The technique is useful only with Ss who manifest definite sequential patterns in choosing stimuli, e.g., either stimulus alternation or stimulus repetition, and it may be limited to two-choice situations.

\section{REFERENCES}

DEMBER, W. N., \& FOWLER, H. Spontaneous alternation behavior. Psychological Bulletin, 1958, 55. $412-428$.

HARRIS, $L$. The effects of relative novelty on children's choice behavior. Joumal of Experimental Child Psychology, 1965, 2, 297-305.

HARRIS, L Effects of amount of relative novelty on children's choice behavior. Psychonomic Science, 1967, 8, 319-320. PETERSON, L. R., SALTZMAN, D. HILLNER, K. \& LAND, V. Recency and frequency in paired-associate learning. Journal of Experimental Psychology, 1962,63, 396-403.

RABINOWITZ. F. M., \& DeMYER, S. Stimulus and response alternation in young children. Developmental Psychology, in press.

SCHEHFE, H. Ine analy sis of variance. New York: Wiley, 1959 